\section{Digestive Diseases}

Atreya, R. 386

Bernstein, C.N. 406

Binion, D.G. 452

Blumberg, R.S. 395

Braun, A. 490

Bruining, D.H. 429

Cima, R.R. 501

Cosnes, J. 411

Dotan, I. 418

Dubinsky, M. 424

Ehehalt, R. 490

Feuerbach, S. 433
Hanauer, S.B. 497

Hanemann, A. 490

Herfarth, H.H. 439

Higgins, P.D.R. 483

Isaacs, K.L. 548

Kane, S. 478

Karner, M. 490

Kaser, A. 395

Khokhar, O.S. 507

Kiesslich, R. 445

Kotton, C.N. 525

Lewis, J.H. 507
Long, M.D. 439

Neurath, M.F. 386

Peyrin-Biroulet, L. 463

Rogler, G. 556

Sandborn, W.J. 536

Schölmerich, J. 472

Siegel, C.A. 543

Stremmel, W. 490

Swoger, J.M. 452

Vavricka, S.R. 556

Weinstock, D.M. 519

Zeissig, S. 395

\title{
Subject Index Vol. 28, No. 3, 2010
}

Adalimumab 497, 536

Adherence 478, 483

Aminosalicylate 478

Anti-glycan antibodies 418

Anti-TNF agents 556

ASCA 424

Azathioprine 536

Balloon enteroscopy 439

Biologics 497

Capsule endoscopy 439

Certolizumab pegol 536

Chemokine receptors 386

Chemokines 386

Chromoendoscopy 445

Ciprofloxacin 556

Clostridium difficile 452

Colectomy 501

Crohn's disease 418, 424, 429, 433, 439, $452,536,543,556$

CT enterography 429

Diagnosis 418, 433

Diet 406, 411

Disability 463

Disease activity 433

Drug hypersensitivity 452

Drug-induced liver injury 507
Endoscopy 439

Environment 406

Epstein-Barr virus 519

Fibrostenosing 424

Fistula 556

Hepatosplenic T cell lymphoma 519

Hepatotoxicity 507

Hypercoagulability 452

Immune suppression 472

Immunosuppression 525

Induction 543

Inflammatory bowel disease(s) 386,395 , $424,439,463,472,507,519,525,536$, 548

Infliximab 497, 536

Internal penetrating disease 424

Intestinal microbial flora 406

Lifestyle 411

Lymphoma 519

Maintenance 483

6-Mercaptopurine 536

Mesalamine 478

Metronidazole 556

MRI enterography 433

Mucosal barrier 472, 490

- healing 548

Mucus 490
Non-steroidal anti-inflammatory drugs 452

Pain 452

pANCA 424

Pathophysiology 395

Phosphatidylcholine 490

Physical activity 411

Pouchitis 424

Prognosis 418

Quality of life 463

Remission 543, 548

Serologic markers 418

Sleep deprivation 452

Small bowel imaging 429

Smart biopsies 445

Smoking 411

Stress 406

Surgery 501

Surveillance 445

Thiopurine 519

Tobacco 406

Total proctocolectomy 501

Treatment 472

Ulcerative colitis $424,445,452,478,483$, 490, 497, 536

- -, chronic 501

Vaccine 525

\section{KARGER}

(C) 2010 S. Karger AG, Basel 